\title{
Measurements of Charge Diffusion in Deep-Depletion CCDs by Optical Diffraction
}

\author{
H. Cease, H.T. Diehl, J. Estrada, B. Flaugher and V. Scarpine \\ Fermi National Accelerator Laboratory, \\ Batavia, Illinois 60510, USA
}

(Dated: February 3, 2008)

\begin{abstract}
The charge diffusion is measured in back illuminated, fully depleted, $250 \mu \mathrm{m}$ thick CCDs by imaging the diffraction pattern of a double slit. The CCDs studied are the focal plane detectors for the Dark Energy Camera (DECam) instrument currently under construction for the Dark Energy Survey (DES). The results presented here indicate that the dispersion of charge due to diffusion can be kept below the DES specification ( $\left.\sigma_{d}<7.0 \mu \mathrm{m}\right)$.
\end{abstract}




\section{INTRODUCTION}

Recent advances [1] in CCD technology allow the fabrication of $\approx 300 \mu \mathrm{m}$ thick devices which are fully depleted at relatively low voltages. These CCDs have a significantly higher efficiency in the near-IR and are the optical detectors chosen by several groups building new mosaic cameras for astronomy, such as DECam [2, 3] , SNAP [4] and HyperSuprime [5]. In this work we study charge diffusion for the DECam $[2,6,7]$ detectors. For a general description of CCDs, particularly n-type, see Ref [8]. DECam is the instrument currently being built for the Blanco 4m Telescope at CTIO [9] that will be used for the Dark Energy Survey (DES) and will be available as a facility instrument at CTIO.

The schematic of the devices developed[1] by Lawrence Berkeley National Laboratory (LBNL) that are going to be used in the DECam focal plane is presented in Fig. 1. It is a back illuminated, p-channel CCD thinned to about $250 \mu \mathrm{m}$ thickness and biased to be fully depleted. The charge collected in the depletion region is stored in the buried channels established a few $\mu \mathrm{m}$ away from the the gate electrodes. The holes produced near the back surface must travel the full thickness of the device to reach the potential well. During this transit inside the depletion region, a hole will also move a little perpendicular to the pixels. This is called charge diffusion and it has to be kept under control in order to avoid a significant degradation in the image quality.

The diffusion in thick CCDs has been identified as an important parameter of the performance of these devices and previous work has been done to characterize this effect $[10,11]$. Diffusion is traditionally measured by imaging a small spot of light (similar in size to a CCD pixel), which is focused on the surface of the CCD and then moving it with a high precision over the surface ("spot machine"). The size of the light spot has to be known. The movement has to be controlled in steps much smaller than the dimensions of the pixel. This techinique been successfully been applied for diffusion measurement in optical devices and

infrared sensors. Diffusion has also been measured in previous work by using X-rays [12]. In this work, we present measurements done with an alternative technique that is based on a somewhat simpler optical setup and no precision focusing or moving is required. 


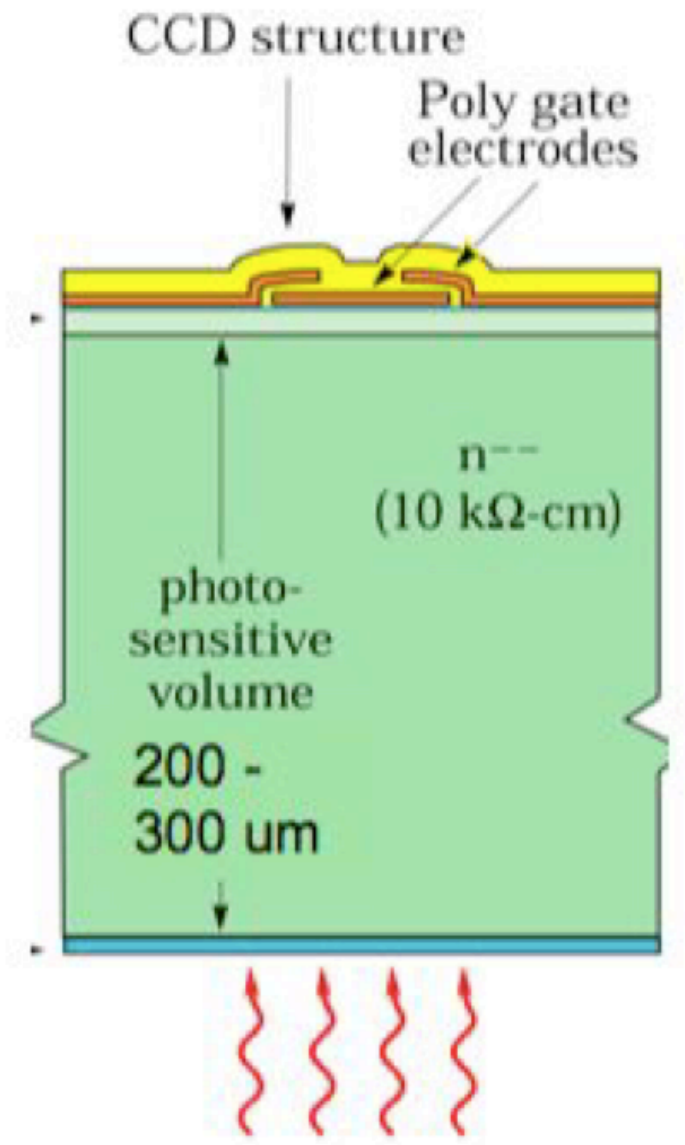

FIG. 1: Schematic of a DECam detector. Back illuminated, $250 \mu \mathrm{m}$ thick, p-channel CCD. For more details see Ref. [1]

\section{EXPERIMENTAL SETUP AND MEASUREMENT}

In this section we describe a method for measuring the diffusion on a CCD detector based on the imaging of a diffraction pattern produced by a double slit (for a general description of optical diffraction see Ref. [13]). As a first step we describe this method using a simulation.

Figure 2 shows a region of the expected diffraction pattern produced by two slits in conditions similar to the ones in our setup: slit width $=0.1 \mathrm{~mm}$, slit spacing $=1 \mathrm{~mm}$, distance from slits to $\mathrm{CCD}=200 \mathrm{~mm}$ ), the effect of pixelation is also included in this simulation. When diffusion is introduced we expect the contrast in the pattern to be reduced as shown in Fig. 3. In order to recover the additional diffusion introduced for each simulation $\left(S_{1}, \ldots, S_{5}\right)$ the Fourier spectra are analyzed. The additional diffusion for $S_{i}$ with respect to $S_{1}\left[\Delta \sigma\left(S_{i}, S_{1}\right)\right]$ corresponds to a convolution of the diffraction pattern with a Gaussian in 


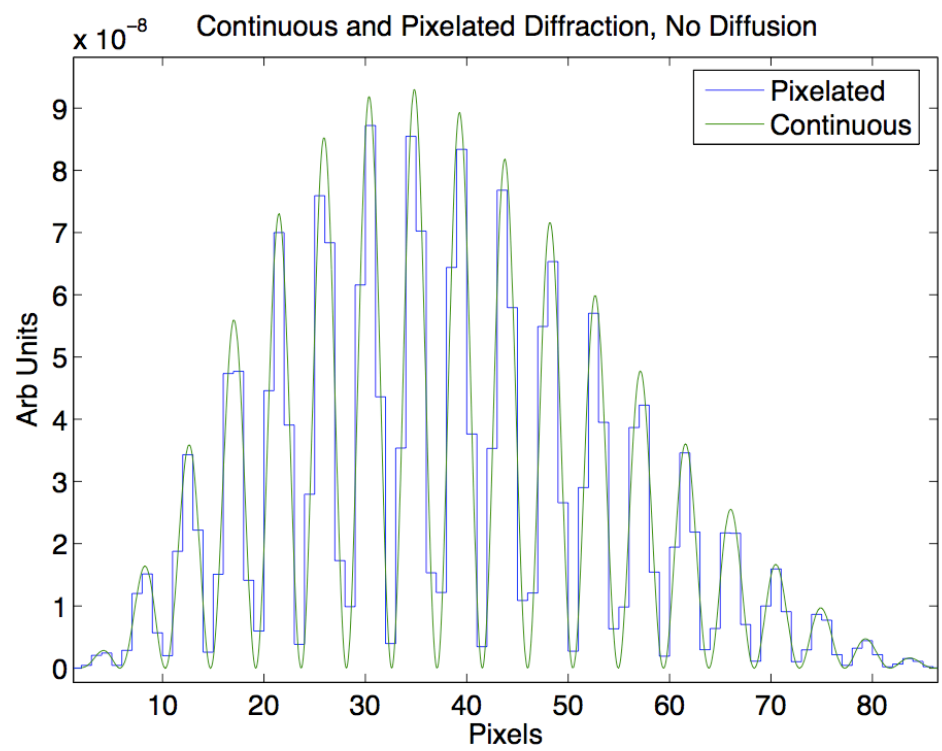

FIG. 2: Simulation of a region of the diffraction pattern expected for a double slit similar to the one used for our experimental setup. This region is away from the center of the pattern where the main intensity peak is located.

physical space, in Fourier space this translates in a product of the Fourier transform of the diffraction patter with a Gaussian (using the convolution theorem). Then, taking the ratio,

$$
\frac{F\left(k_{2}, S_{1}\right) / F\left(k_{1}, S_{1}\right)}{F\left(k_{2}, S_{i}\right) / F\left(k_{1}, S_{i}\right)}=e^{-\frac{\left(k_{2}^{2}-k_{1}^{2}\right) \Delta \sigma^{2}\left(S_{i}, S_{1}\right)}{2}},
$$

where $F(k, S)$ is the value of the Fourier power at spacial frequency $k$ for simulation $S$. We use as $k_{2}=40 \times 2 \pi / 100$ pix (giving a period of 5 pixels), the position of the peak, and $k_{1}=6 \times 2 \pi / 100$ pix as a baseline for the comparison. The results of this analysis indicate that in these conditions, considering the pixelation and doing the Fourier analysis in a finite area, it is possible to recover the simulated diffusion with $1 \%$ precision using this technique (this simulation does not include the noise in the CCD image). In the rest of this work we will apply this method to the measurement of diffusion in DECam CCDs [6].

A typical example of a region of this diffraction pattern is shown in Fig.4. As can be seen in the image, there is a high and low spatial frequency component. The high spatial frequency component corresponds to a spatial period of approximately 5 pixels.

In previous work [1] [10] it has been shown that for these CCDs the spatial variance resulting from diffusion $\left(\sigma_{d}^{2}\right)$ depends on the substrate voltage $\left(V_{\text {sub }}\right)$ used to obtain the depletion. For example if $V_{\text {sub }}$ is reduced, allowing extra diffusion in the CCD, the image 


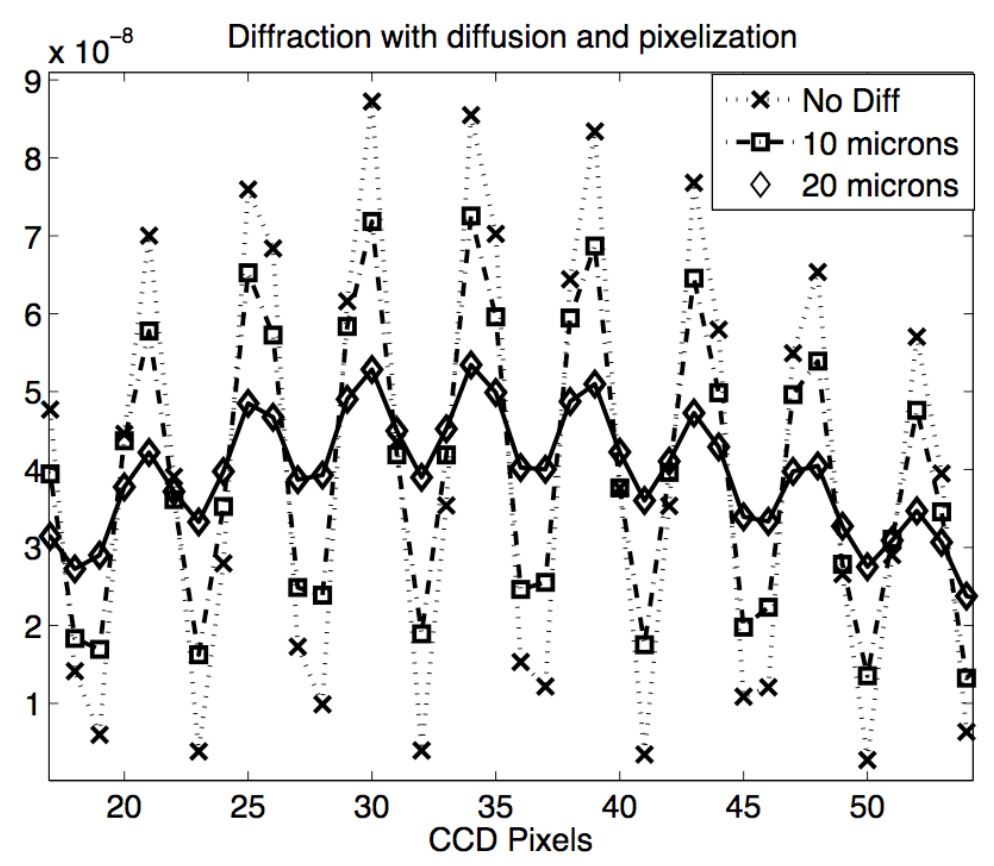

FIG. 3: Simulation of a diffraction slit image with pixelization and different values for diffusion. It is clear from this simulation that the additional diffusion will reduce the contrast in the interference pattern.

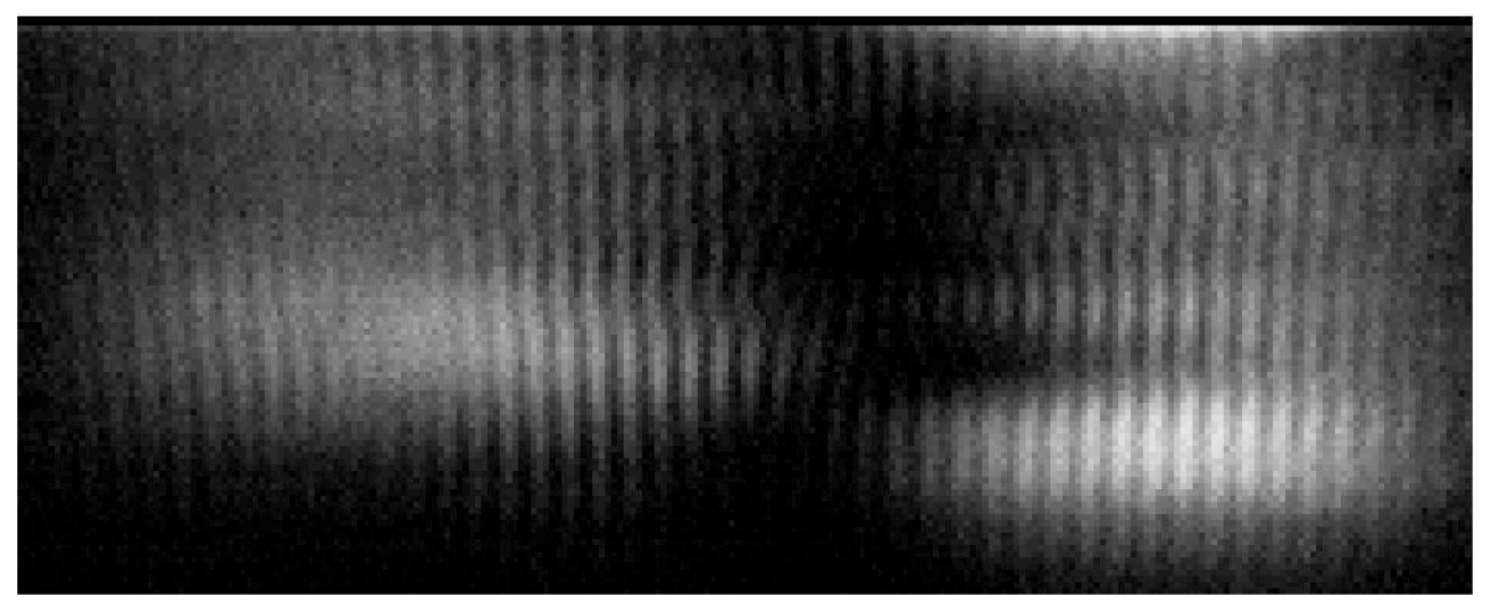

FIG. 4: Double slit diffraction pattern produced with two slits with width $100 \mu \mathrm{m}$ and separated by $1 \mathrm{~mm}$ illuminated with a HeNe laser and imaged with a $2 \mathrm{k}$ x $2 \mathrm{k}$ CCD $200 \mathrm{~mm}$ away from the slits, back illuminated, $250 \mu \mathrm{m}$ thick CCD from the engineering run of DECam CCDs. For this image the CCD was operated with $V_{\text {sub }}=70 \mathrm{~V}$ as discussed in the text. The image shows only a small fraction of the full pattern, corresponding to the region used for the analysis described below. 


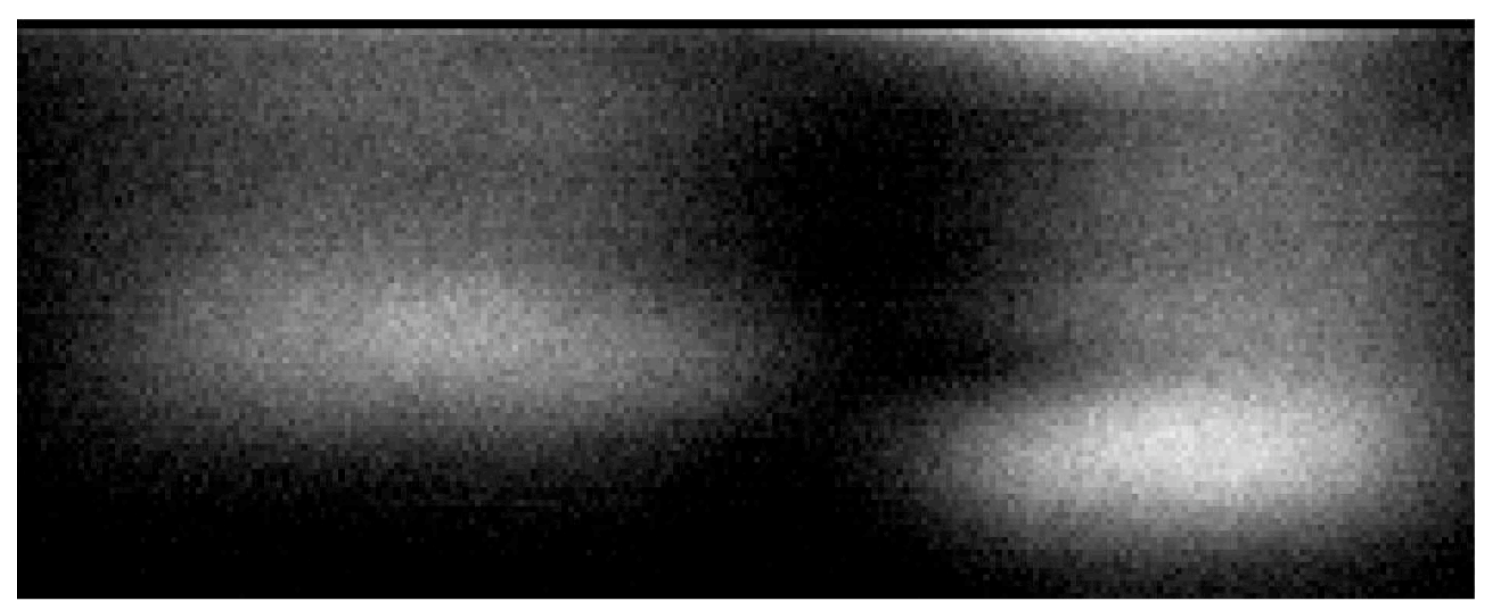

FIG. 5: Same as Fig. 4, but now reducing the substrate voltage to $V_{\text {sub }}=10 \mathrm{~V}$.

smears making the high spatial frequency component weaker, as shown in Fig. 5. By measuring the difference in the Fourier power spectrum between Fig.4 and Fig.5, one can quantify the increase in the diffusion effect produced by the reduction in $V_{s u b}$.

The Fourier power spectrum for Fig. 4 with $V_{\text {sub }}=70 \mathrm{~V}$ is compared to that of Fig.5 with $V_{\text {sub }}=10 \mathrm{~V}$, as shown in Fig.6. From the plot is clear that the high spatial frequency component $(k \approx 40)$ is completely smeared at $V_{\text {sub }}=10 \mathrm{~V}$. The additional diffusion $\Delta \sigma\left(V_{B}, V_{A}\right)$ introduced by the reduction of $V_{\text {sub }}$ from $V_{B}$ to $V_{A}$ can be quantified by looking at the difference between the two Fourier spectra. In $k$ space, the additional diffusion translates into a multiplication of the Fourier spectrum by a Gaussian with width $1 / \Delta \sigma\left(V_{B}, V_{A}\right)$,

$$
F\left(k ; V_{A}\right) \propto F\left(k ; V_{B}\right) e^{-\frac{\left[k \Delta \sigma\left(V_{B}, V_{A}\right)\right]^{2}}{2}},
$$

where $F\left(k ; V_{\text {sub }}\right)$ is the Fourier spectrum for the spatial frequency $k$ and substrate voltage $V_{\text {sub }}$. Given two spatial frequencies $k_{1}$ and $k_{2}$ (with $k_{2}>k_{1}$ ), $\Delta \sigma\left(V_{B}, V_{A}\right.$ ) is extracted from the relation

$$
\frac{F\left(k_{2}, V_{A}\right) / F\left(k_{1}, V_{A}\right)}{F\left(k_{2}, V_{B}\right) / F\left(k_{1}, V_{B}\right)}=e^{-\frac{\left(k_{2}^{2}-k_{1}^{2}\right) \Delta \sigma^{2}\left(V_{B}, V_{A}\right)}{2}} .
$$

In principle $k_{2}$ and $k_{1}$ could be any frequencies. The double slit diffraction pattern shown in Fig. 4 produces a peak at high spacial frequencies $(k=40 \times 2 \pi / 100$ pix $)$ corresponding to a period of 5 pixels), the high signal to noise for the spectrum around this peak makes it a good selection for $k_{2}$. On the other hand, $k_{1}$ is only used for the normalization of the spectrum and the detailed selection of this point is not critical for our measurement, we used $k_{1}=6 \times 2 \pi / 100$ pix. 


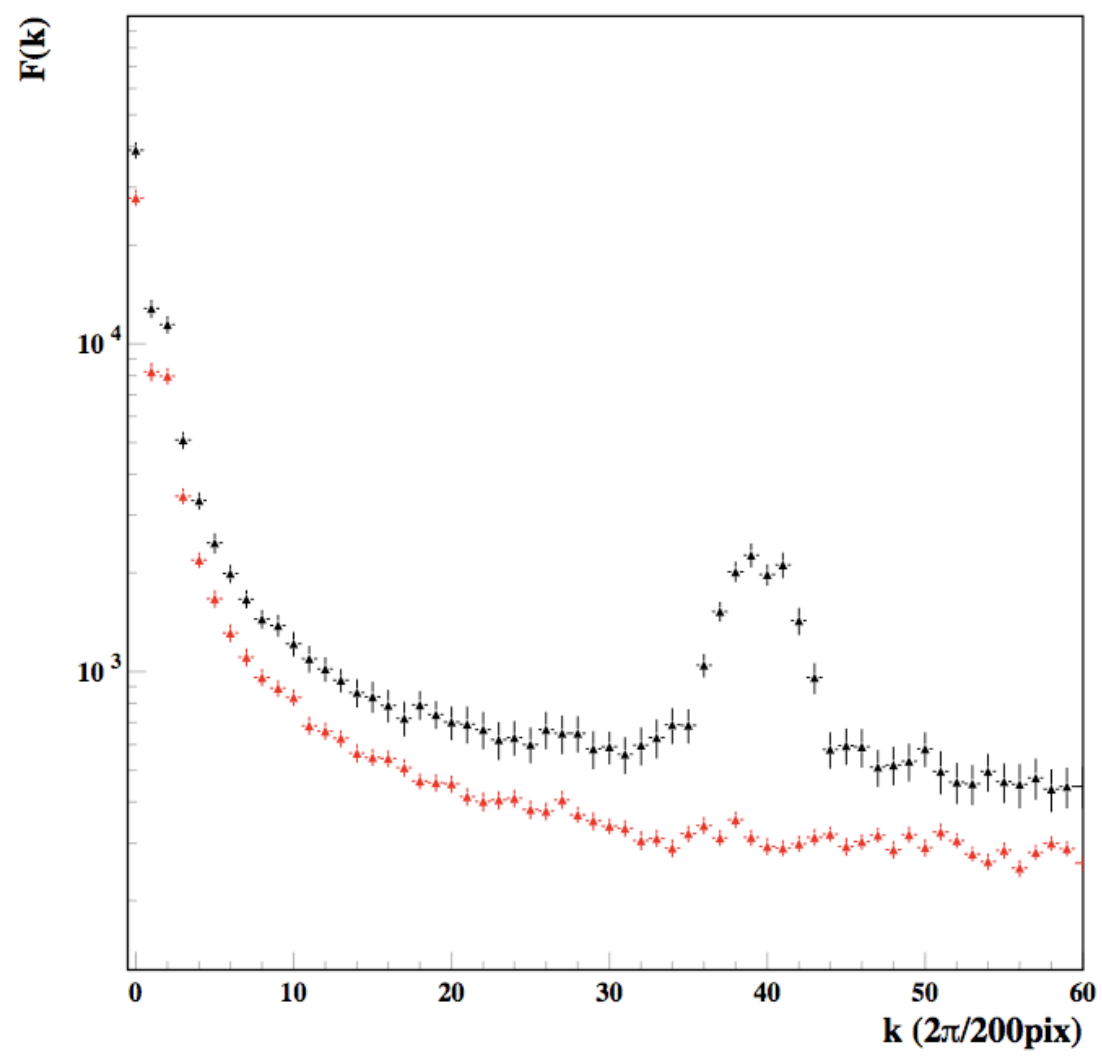

FIG. 6: Fourier spectrum for Fig. 4 (black) and Fig. 5 (red). The data corresponding to low substrate voltage(red) does not have a significant peak at high spatial frequencies.

Using Eq. (3), $\Delta^{2} \sigma\left(V_{\text {sub }}=70 \mathrm{~V}, V_{A}\right)$ was measured over a wide range of $V_{\text {sub }}$ for the CCD shown in Figs. 4 and 5 . The resulting values for $\Delta^{2} \sigma\left(V_{\text {sub }}=70 \mathrm{~V}, V_{A}\right)$ are presented in Fig.7.

As shown in Ref. [1], when $V_{\text {sub }}$ is sufficiently high (overdepleted CCD) the diffusion is

$$
\sigma_{d}^{2} \propto 1 /\left(V_{s u b}-V_{j}\right)
$$

where $V_{j}$ is the junction, such that voltage $\left(V_{s u b}-V_{j}\right)$ is the voltage drop across the depletion region. Eq. (4) can be expressed as

$$
\sigma_{d}^{2}\left(V_{A}\right)=\sigma_{d}^{2}\left(V_{\text {sub }}=70 \mathrm{~V}\right)+\Delta^{2} \sigma\left(V_{\text {sub }}=70 \mathrm{~V}, V_{A}\right)=\frac{\alpha}{V_{A}-V_{j}},
$$

where $\alpha$ is depends on temperature, drift distance and charge of the carries [1]. This translates into the relation

$$
\Delta^{2} \sigma\left(V_{\text {sub }}=70 V, V_{A}\right)=\frac{\alpha}{V_{A}-V_{j}} m(T, E)-\sigma_{d}^{2}\left(V_{\text {sub }}=70 \mathrm{~V}\right) .
$$


where the $m(T, E)$ is a factor taking into account the mobility dependence on temperature and electric field $E$ discussed in Ref. [10] ,

$$
m(T, E)=\left[1+\left(<E>/ E_{c}\right)^{\beta}\right]^{1 / \beta} .
$$

With $\beta=0.46 T^{0.17}, E_{c}=1.24 T^{1.68} \mathrm{~V} / \mathrm{cm}$ and $\langle E>$ being the mean electrical field in the depletion region. This means that the diffusion at $V_{\text {sub }}=70 \mathrm{~V}$ can be obtained from a fit between $\Delta^{2} \sigma\left(V_{\text {sub }}=70 \mathrm{~V}, V_{A}\right)$ and $V_{A}$, as shown in Fig.7. The results of this fit, combined with Eq.(5) are used to covert the $\Delta^{2} \sigma\left(V_{\text {sub }}=70 \mathrm{~V}, V_{A}\right)$ measurements into an absolute diffusion measurement. The data collected for this diffusion measurement does not have the power of determining $V_{j}$. To demonstrate this, we fitted $\alpha$ and $\sigma_{d}^{2}\left(V_{s u b}=70 \mathrm{~V}\right)$ after fixing $V_{j}=-25 \mathrm{~V},-24 \mathrm{~V},-23 \mathrm{~V}, \ldots,-5 \mathrm{~V}$ (from Ref. [10], we expect $V_{j}=? 13 \mathrm{~V}$ ), the results are shown in Fig. 7. From the plot, it is not possible to separate the curves fitted with different values of $V_{j}$, indicating that the presented data can not distinguish between these values. For this reason, we allow $V_{j}$ to vary in the range $\left(-25 \mathrm{~V}<V_{j}<-5 \mathrm{~V}\right)$ and fit $\sigma_{d}^{2}\left(V_{s u b}=70 \mathrm{~V}\right)$ for each value of $V_{j}$. As a result we obtain $\tilde{\sigma}_{d}^{2}\left(V_{\text {sub }}=70 \mathrm{~V}\right)$ as the average for $\sigma_{d}^{2}\left(V_{\text {sub }}=70 \mathrm{~V}\right)$ in the allowed range with an uncertainty corresponding to the variation of $\sigma_{d}^{2}\left(V_{\text {sub }}=70 \mathrm{~V}\right)$ in that range (we effectively marginalize over $V_{j}$ in this way) . The results in Table I show the $\alpha$ and $\sigma_{d}^{2}\left(V_{s u b}=70 \mathrm{~V}\right)$ values obtained for $V_{j}=-15 \mathrm{~V}$ and the $\sigma_{d}^{2}\left(V_{\text {sub }}=70 \mathrm{~V}\right)$ with the corresponding uncertainty. We use $\tilde{\sigma}_{d}^{2}\left(V_{\text {sub }}=70 \mathrm{~V}\right)$ in Eq.(5) to obtain values for absolute diffusion.

\section{RESULTS AND CONCLUSION}

The results presented in Fig. 8 corresponds to three 2k x 2k CCDs from DECam engineering production runs and are compared with results published in previous work [10] obtained for a SNAP[4] CCD. The DECam CCDs have been certified to support up to $V_{\text {sub }}=40 \mathrm{~V}$, the long term performance of the devices could be compromised at higher voltages. The tests done for this work included measurements with $V_{s u b}>40 \mathrm{~V}$, but these higher voltages were applied to the device only for a short time (a few minutes). The results obtained indicate that for $V_{\text {sub }}=40 \mathrm{~V}$ the DES CCDs will have a dispersion due to charge diffusion of $\sigma_{d}<7.5 \mu \mathrm{m}$, as established in the DES technical requirement. Table I shows a summary of the results obtained at $V_{\text {sub }}=40 \mathrm{~V}$. 


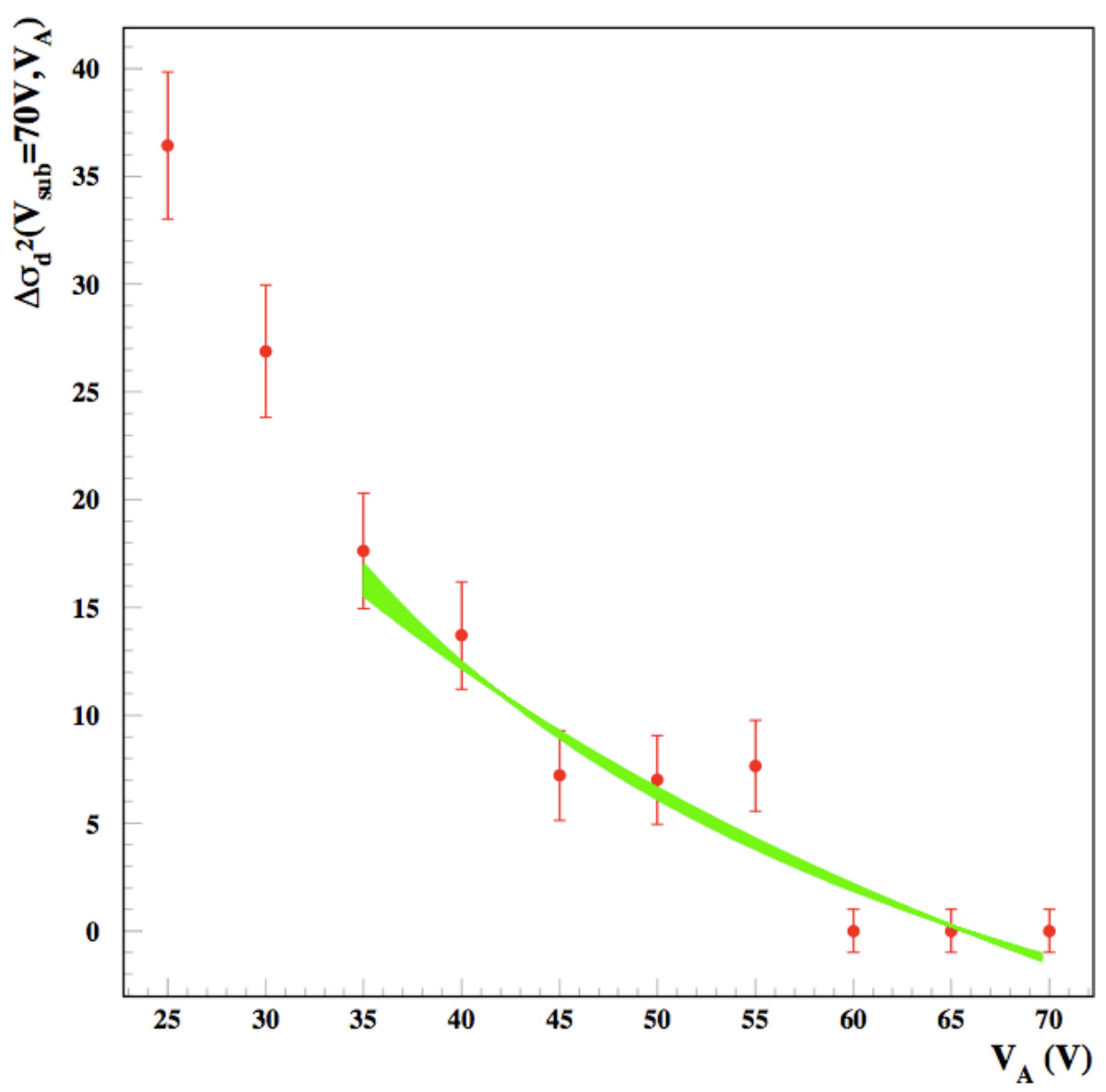

FIG. 7: Variance introduced by the diffusion as a function of $1 / V_{\text {sub }}$. The curve corresponds to Eq.(6). The width of the curve is produced by varying the value of $V_{j}$ in the range $-25 \mathrm{~V}<V_{j}<-5$ V.

In summary, a new method for measuring diffusion in thick CCDs based on the imaging of a double slit diffraction pattern was presented. This technique simplifies significantly the setup needed for the measurement with respect to the more traditional "spot machine" method and will permit the characterization of the diffusion in a large number of the DECam CCDs. However, the new technique is not capable of making a localized measurement of diffusion in a very small area of the sensor, this measurement can be done with the more sophisticated setup corresponding to the "spot machine". By changing the wavelength of the illumination source this method allows for the measurement of the diffusion at different wavelengths. The new technique uses the theoretical prediction for the relation between the substrate voltage and the diffusion to produce a diffusion measurement. 


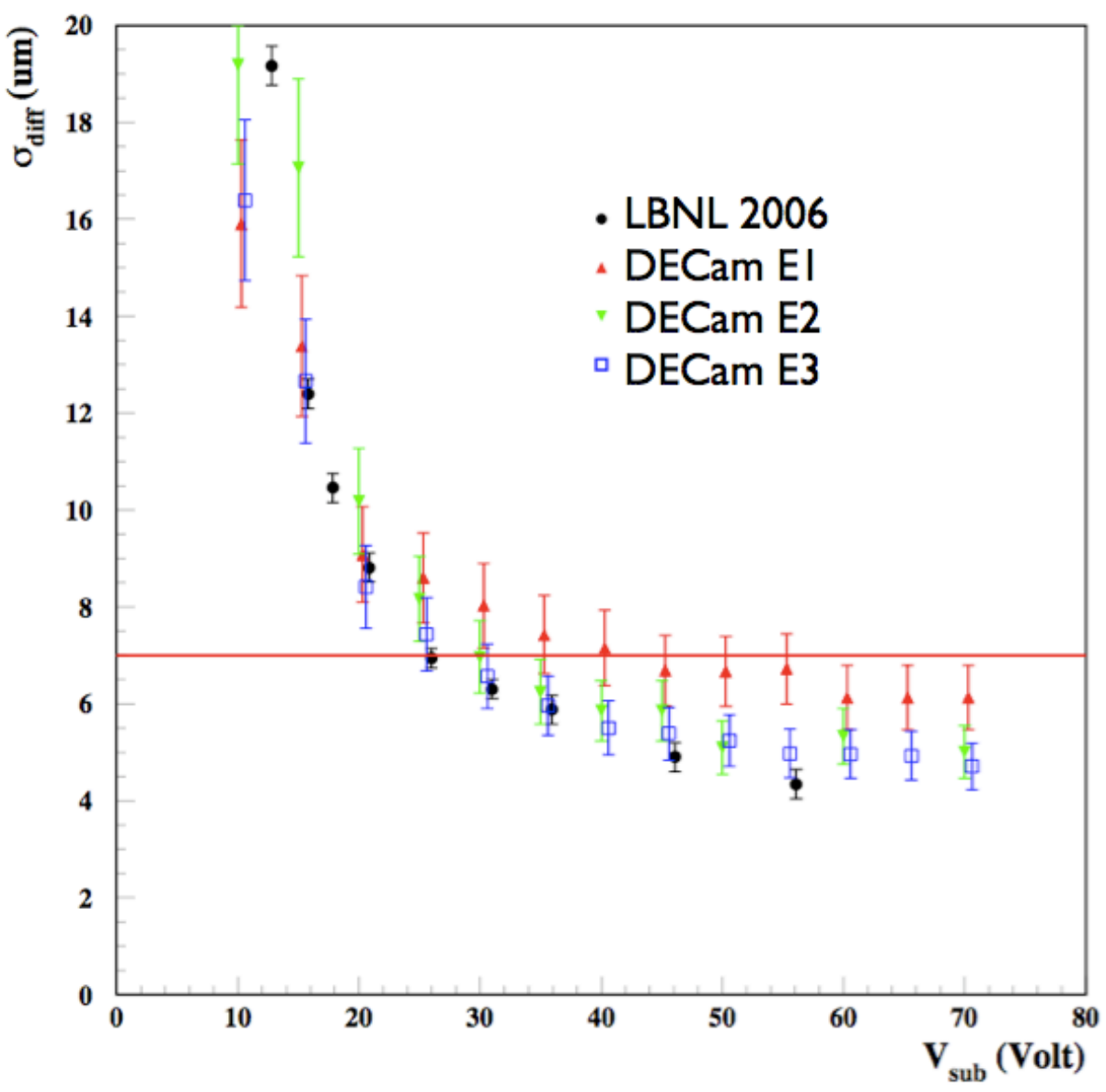

FIG. 8: Result of dispersion due to charge diffusion measured for three $2 \mathrm{k} \mathrm{x} 2 \mathrm{k}, 250 \mu \mathrm{m}$ thick CCD from the engineering production run for DECam. The plot also shows the results obtained in previous work [10] using the "spot machine" on devices similar to those for DECam CCDs, but $200 \mu \mathrm{m}$ thick (black circles). The horizontal line corresponds to a diffusion of $7 \mu \mathrm{m}$.

The optical design team from DECam [14] has established a specification of $\sigma_{d}<7.5 \mu \mathrm{m}$ coming from the scientific requirements for the DES survey. The results presented here show that the fabricated detectors meet this specification.

\section{Acknowledgments}

We thank the LBNL CCD group for reading this manuscript and contributing with insightful comments and suggestions for the article, specially: DonGroom, NatalieRoe, Kyle Dawson and Steve Holland. We thank the CCD testing team at Fermilab for their collab- 


\begin{tabular}{|c|c|c|c|c|c|c|}
\hline $\mathrm{CCD}$ & $\sigma_{d}(\mu \mathrm{m})$ & thickness $(\mu \mathrm{m})$ & method & $\sigma^{2}(70 \mathrm{~V})\left(\mu \mathrm{m}^{2}\right)$ & $\alpha\left(\mu \mathrm{m}^{2} / \mathrm{V}\right)$ & $\tilde{\sigma}^{2}(70 \mathrm{~V})\left(\mu \mathrm{m}^{2}\right)$ \\
\hline \hline DEcam E1 & $6.1 \pm 1.2$ & $250($ DECam $)$ & double slit & $24.9 \pm 4.8$ & $1491 \pm 253$ & $25.0 \pm 5.4$ \\
DEcam E2 & $7.2 \pm 0.8$ & $250($ DECam $)$ & double slit & $37.3 \pm 4.5$ & $2197 \pm 249$ & $37.5 \pm 8.1$ \\
DEcam E3 & $5.8 \pm 1.0$ & $250($ DECam $)$ & double slit & $22.0 \pm 7.0$ & $1359 \pm 394$ & $22.2 \pm 4.5$ \\
\hline \hline
\end{tabular}

TABLE I: Diffusion measurements for the three DECam engineering CCDs (E1,E2 and E3 as shown in Fig. 8. The measurements correspond to $V_{\text {sub }}=40 \mathrm{~V}$.

oration taking these data and their work in the infrastructure needed in this project: K. Kuk, T. Shaw, K. Schultz, W. Stuermer and M. Watson.

[1] S.E. Holland, D.E. Groom, N.P. Palaio, R. J. Stover, and M. Wei, IEEE Trans. Electron Dev. 50 (3), 225-238 (January 2003) LBNL-49992.

[2] Flaugher, B., Ground-based and Airborne Instrumentation for Astronomy. Edited by McLean, Ian S.; Iye, Masanori. Proceedings of the SPIE, Volume 6269, (2006)

[3] Dark Energy Survey Collaboration, astro-ph/0510346.

[4] "Supernova / Acceleration Probe: A Satellite Experiment to Study the Nature of the Dark Energy", SNAP Collaboration, G. Aldering et al., submitted to Publ. Astr. Soc. Pac., astroph/0405232; SNAP Collaboration, astro-ph/0507459.

[5] M. Satoshi et al., Ground-based and Airborne Instrumentation for Astronomy. Edited by McLean, Ian S.; Iye, Masanori. Proceedings of the SPIE, Volume 6269, (2006)

[6] J. Estrada \& R. Schmidt, Scientific Detectors for Astronomy 2005, Edited by J.E. Beletic, J.W. Beletic and P. Amico, Springer, (2005).

[7] J. Estrada et al. , Ground-based and Airborne Instrumentation for Astronomy. Edited by McLean, Ian S.; Iye, Masanori. Proceedings of the SPIE, Volume 6269, (2006).

[8] J.R. Janesick, Scientific Charge Cupled Devices, SPIE press (2001).

[9] T. M. C. Abbott et al. ,Ground-based and Airborne Instrumentation for Astronomy. Edited by McLean, Ian S.; Iye, Masanori. Proceedings of the SPIE, Volume 6269, (2006)

[10] J.A. Fairfield, D. E. Groom, S. J. Bailey, C. J. Bebek, S. E. Holland, A. Karcher, W. F. 
Kolbe, W. Lorenzon, \& N. A. Roe, Fairfield IEEE Trans. Nucl. Sci. 53 (6), 3877-3881 (2006)

[11] A. Karcher, C.J. Bebek, W. F. Kolbe, D. Maurath, V. Prasad, M. Uslenghi, M. Wagner, IEEE Trans. Nucl. Sci. 51 (5), (2004) LBNL-55685.

[12] S.A. Rodney \& J.L. Tonry, astro-ph/0604322.

[13] F.S.Crawford, McGraw-Hill, 1968.

[14] S. Kent et al. ,Ground-based and Airborne Instrumentation for Astronomy. Edited by McLean, Ian S.; Iye, Masanori. Proceedings of the SPIE, Volume 6269, (2006) 